\title{
Novel KCNQ3 Mutation in a Large Family with Benign Familial Neonatal Epilepsy: A Rare Cause of Neonatal Seizures
}

\author{
Snezana Maljevic ${ }^{a, f}$ Sabina Vejzovic ${ }^{a}$ Matthias K. Bernhard ${ }^{c} \quad$ Astrid Bertsche $^{c}$ \\ Sebastian Weise $^{c}$ Miriam Döcker ${ }^{b}$ Holger Lerche ${ }^{a} \quad$ Johannes R. Lemke ${ }^{d}$ \\ Andreas Merkenschlager ${ }^{c}$ Steffen Syrbe ${ }^{c, e}$

\begin{abstract}
aDepartment of Neurology and Epileptology, Hertie Institute for Clinical Brain Research, University of Tübingen, and and Inherited Metabolic Diseases, Department of General Pediatrics, Center for Pediatrics and Adolescent Medicine, University Hospital Heidelberg, Heidelberg, Germany; ${ }^{f}$ The Florey Institute of Neuroscience and Mental Health, Melbourne, Vic., Australia
\end{abstract} \\ ${ }^{b} \mathrm{CeGaT} \mathrm{GmbH}$, Tübingen, ' Department of Women and Child Health, Hospital for Children and Adolescents, and \\ dInstitute of Human Genetics, University of Leipzig Hospitals and Clinics, Leipzig, and ${ }^{\circ}$ Division of Child Neurology
}

\section{Key Words}

Familial neonatal convulsions - KCNQ2 - KCNQ3 ·

Levetiracetam · Neonatal seizures · Potassium channels

\begin{abstract}
Benign familial neonatal seizures (BFNS) present a rare familial epilepsy syndrome caused by genetic alterations in the voltage-gated potassium channels Kv7.2 and Kv7.3, encoded by KCNQ2 and KCNQ3. While most BFNS families carry alterations in KCNQ2, mutations in KCNQ3 appear to be less common. Here, we describe a family with 6 individuals presenting with neonatal focal and generalized seizures. Genetic testing revealed a novel $K C N Q 3$ variant, c.835G $>$ T, cosegregating with seizures in 4 tested individuals. This variant results in a substitution of the highly conserved amino acid valine localized within the pore-forming transmembrane segment S5 (p.V279F). Functional investigations in Xenopus laevis oocytes revealed a loss of function, which supports p.V279F as a pathogenic mutation. When p.V279F was coexpressed with the wild-type (WT) Kv7.2 subunits, the resulting potassium currents were about 10 -fold reduced com-
\end{abstract}

\section{KARGER}

E-Mail karger@karger.com

www.karger.com/msy pared to the WT Kv7.3 and Kv7.2 coexpression. Genotypephenotype correlation shows an incomplete penetrance of p.V279F. Response to antiepileptic treatment was variable, but evaluation of treatment response remained challenging due to the self-limiting character of the disease. The identification of the pathogenic variant helped to avoid unnecessary investigations in affected family members and allowed guided therapy.

(c) 2016 S. Karger AG, Basel

Benign familial neonatal epilepsy (BFNS1/2) is a rare inherited epilepsy disorder (OMIM 121200 and 121201) first described by Rett and Teubel [1964]. Mutations in the 2 genes KCNQ2 in chromosome 20q and KCNQ3 in chromosome 8q encoding the voltage-gated potassium channel subfamily KQT members 2 (Kv7.2) and 3 (Kv7.3) have been reported to result in BFNS [Biervert et al., 1998, Charlier et al., 1998, Singh et al., 1998]. KCNQ2, KCNQ3, and KCNQ5 encode subunits of the widely expressed homo- and heterotetrameric M-channels, yielding the slowly activating and deactivating potassium M-current, 
which can be blocked by muscarinic agonists [Maljevic and Lerche, 2014]. In $>95 \%$ of all cases, BFNS can be attributed to mutations in KCNQ2. Only a few mutations in $K C N Q 3$ have so far been associated with this disorder and associated electrophysiological data is scarce (HGMD, http://www.hgmd.cf.ac.uk/) [Singh et al., 2003, Zara et al., 2013, Maljevic and Lerche, 2014].

Our study aimed to report phenotypic data on members of a BFNS family carrying a novel missense mutation in $K C N Q 3$ and to investigate the impact of the mutation on channel function.

\section{Methods}

\section{Genetic Testing}

Genomic DNA of the index patient (IP) and available family members was extracted from peripheral blood lymphocytes. The coding exons of KCNQ2 (accession number hg19: NM 172108.3) and KCNQ3 (accession number hg19: NM 004519.2) were sequenced by conventional Sanger sequencing. Deletion/duplication screening was performed by MLPA. Predictions of the functional impact of identified coding variants were assessed by different in silico analysis programs (PolyPhen2, http://www.genetics.bwh. harvard.edu/pph2 and MutationTaster, http://www.mutationtaster.org). Variants were compared to 60,706 controls of the ExAC browser (http://exac.broadinstitute.org/) and in-house data. Conservation of mutated positions was evaluated using sequence alignment of different species and different human Kv-channels.

\section{Functional Studies}

Mutagenesis and RNA Preparation

The Quick Change kit (Stratagene, La Jolla, Calif., USA) was used to engineer the missense mutation in the KCNQ3 cDNA inserted into the pTLN vector. The mutation was confirmed and additional mutations excluded by Sanger sequencing. Primers are available upon request. cRNA was prepared using the SP6 mMessage mMachine kit from Ambion (Agilent Technologies, Santa Clara, Calif., USA).

Oocyte Preparation and Injection

Oocytes were obtained from the Institute of Physiology I, Tübingen and prepared as previously described [Orhan et al., 2014]. The concentrations of Kv7.2 and Kv7.3 mutant and wildtype (WT) cRNAs were adjusted to the same value and cRNA mixtures in appropriate ratios injected in parallel using Robooinject ${ }^{\circledR}$ (Multi Channel Systems, Reutlingen, Germany). The volume of injected cRNA was kept constant (50 nl).

Automated Oocyte 2-Microelectrode Voltage Clamp

Potassium currents in oocytes were recorded 2-3 days after injection using Roboocyte $2^{\circledR}$ (Multi Channel Systems, Reutlingen, Germany). The intracellular glass microelectrodes had a resistance of $0.3-1 \mathrm{M} \Omega$ when filled with $1 \mathrm{M} \mathrm{KCl} / 1.5 \mathrm{M} \mathrm{KAc}$. The bath solution was $\mathrm{ND} 96$ (in mM: $93.5 \mathrm{NaCl}, 2 \mathrm{KCl}, 1.8 \mathrm{CaCl}_{2}, 2 \mathrm{MgCl}_{2}$, and 5 Hepes; pH 7.5). Currents were sampled at $1 \mathrm{kHz}$. To analyze channel activation, we used 2 -second long depolarizing steps $(\Delta 10$
$\mathrm{mV}$ ) from a holding potential of -80 to $+40 \mathrm{mV}$, followed by a step to $-30 \mathrm{mV}$ for $0.5 \mathrm{~s}$ to analyze the tail currents [Orhan et al., 2014].

\section{Data Analysis}

Voltage clamp recordings were analyzed using Roboocyte2+ (Multi Channel Systems), Clampfit (pClamp 8.2, Axon Instruments, Union City, Calf., USA), Microsoft Excel (Microsoft, Redmond, Wash., USA), and GraphPad Prism (GraphPad Software, La Jolla, Calif., USA). Maximum current amplitudes were compared at the end of a 2 -second test pulse to $+40 \mathrm{mV}$. The voltage dependence of channel activation was derived from tail current amplitudes recorded at $-30 \mathrm{mV}$. A Boltzmann function was fit to the current-voltage relationships, $\mathrm{I}(\mathrm{V})=\operatorname{Imax} /\left(1+\exp \left[\left(\mathrm{V}-\mathrm{V}_{0.5}\right) /\right.\right.$ $\mathrm{k}])+\mathrm{C}$, where Imax is the maximum tail current amplitude at test potential $\mathrm{V}, \mathrm{V}_{0.5}$ the half-maximal activation potential, $\mathrm{k}$, a slope factor reflecting characteristics of voltage-dependent channel gating and $\mathrm{C}$, a constant. All data are shown as mean values \pm SEM. Statistical significance was evaluated using one-way ANOVA with Dunnett's post hoc test. Multiple comparisons were performed to Kv7.2/Kv7.3 channels as a control (GraphPad Prism).

\section{Results}

\section{Clinical Features}

The IP (IV.2), a 6-day-old eutrophic boy, born at term, was admitted to hospital because of a focal clonic, secondarily generalized tonic-clonic seizure with cyanosis lasting $1 \mathrm{~min}$. Repetitive focal clonic seizures, involving legs and arms asymmetrically, had been observed from the day before. After admission, frequent generalized seizures with oxygen desaturation to $40 \%$ were observed, necessitating intensive care unit monitoring. Extensive investigations including infectiological, metabolic screenings (electrolytes, glucose, ammonia, acylcarnitines, very long-chain fatty acids, cerebrospinal fluid parameters, lactate, pterines, neurotransmitter, amino acids, pipecolic acid, urinary organic acids, sulfite test, and creatine metabolism) was well as brain MRI on postnatal day 6 (d6) gave normal results. Electroencephalography (EEG) repeatedly displayed focal epileptic discharges and clonic seizures of focal origin (fig. 1). Up to 5 seizures per day occurred until d20 with only 1 seizure on d18 and d19. After trials with pyridoxine and pyridoxal-phosphate, levetiracetam (LEV) was added on d8 with increasing doses reaching $65 \mathrm{mg} / \mathrm{kg}$ body weight on $\mathrm{d} 20$. The patient was discharged in good clinical condition on $\mathrm{d} 25$. Treatment with LEV was successfully discontinued after 8 months of age. The IP is now 4 years old and shows an age-appropriate development. Two first cousins of the IP (IV.4 and IV.5) were identified and treated after the identification of the pathogenic variant in this family. Both cousins received only basic investigations to rule out other causes of neonatal seizures. Pro- 
Fig. 1. Ictal Video-EEG recording of the IP on postnatal $\mathrm{d} 7$, documenting right hemispheric onset of a focal seizure (A) with upward gaze to the left side, hemifacial twitching (B) and clonus of the left hand (not seen). Secondarily generalized rhythmic spike-wave activity (C), clinically accompanied by apnea and cyanosis is followed by generalized slowing of the background activity (D).

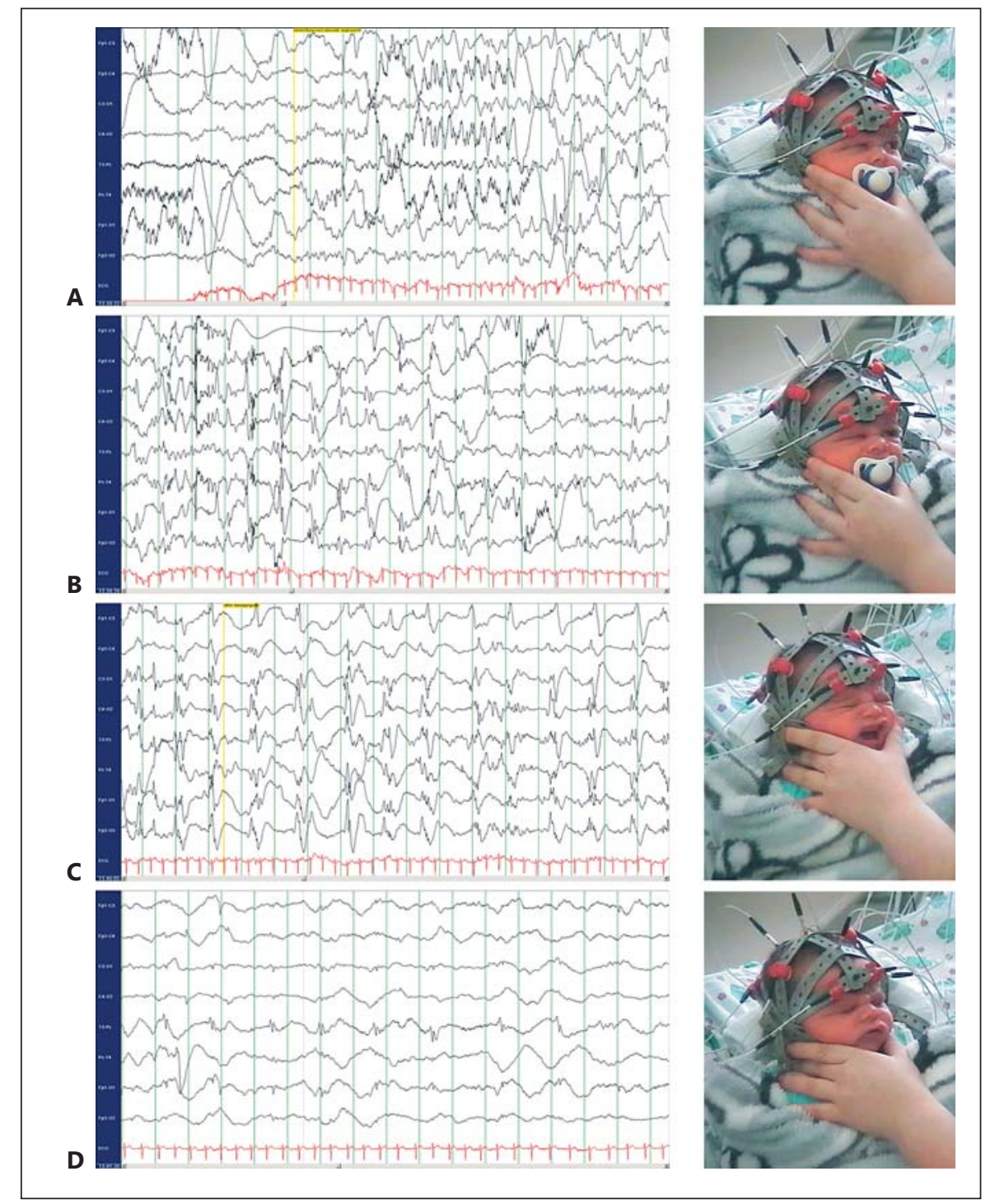

longed cyanotic seizures prompted anticonvulsant treatment in both. While IV.4 became seizure free after introduction of LEV on d12, seizures persisted despite LEV treatment (up to $85 \mathrm{mg} / \mathrm{kg} / \mathrm{d}$ ) in patient IV.5. He eventually became seizure free after switching to oxcarbazepine (OXC) after d10. The pedigree analysis (fig. 2) of the family revealed self-limited cyanotic neonatal seizures that prompted hospitalization in 3 more relatives, starting between $\mathrm{d} 2$ and $\mathrm{d} 4$. None of the affected individuals displayed seizures after the first month of life, independent of anticonvulsant treatment, and all showed a normal psychomotor development later in life. Clinical information is provided in table 1 for all affected family members where data was available.

\section{Genetic Testing}

In the IP, we detected the intronic KCNQ2 variant c.297-18C>T and the KCNQ3 missense variant c.835G $>$ T, p.V279F. KCNQ2 c. $297-18 \mathrm{C}>\mathrm{T}$ is repeatedly seen in controls in the ExAC database (56/60.706), whereas KCNQ3 p.V279F is absent. Standard in silico prediction tools (PolyPhen2 and MutationTaster) predicted KCNQ2 c.297-18C>T as benign and KCNQ3 p.V279F as likely pathogenic with a very high confidence score (>0.999). Overall 6 affected individuals and 2 unaffected obligate carriers were identified within this family. Further segregation analysis confirmed the presence of the KCNQ3 p.V279F in 4 more affected individuals and obligate carriers while KCNQ2 c.297-18C>T did not 


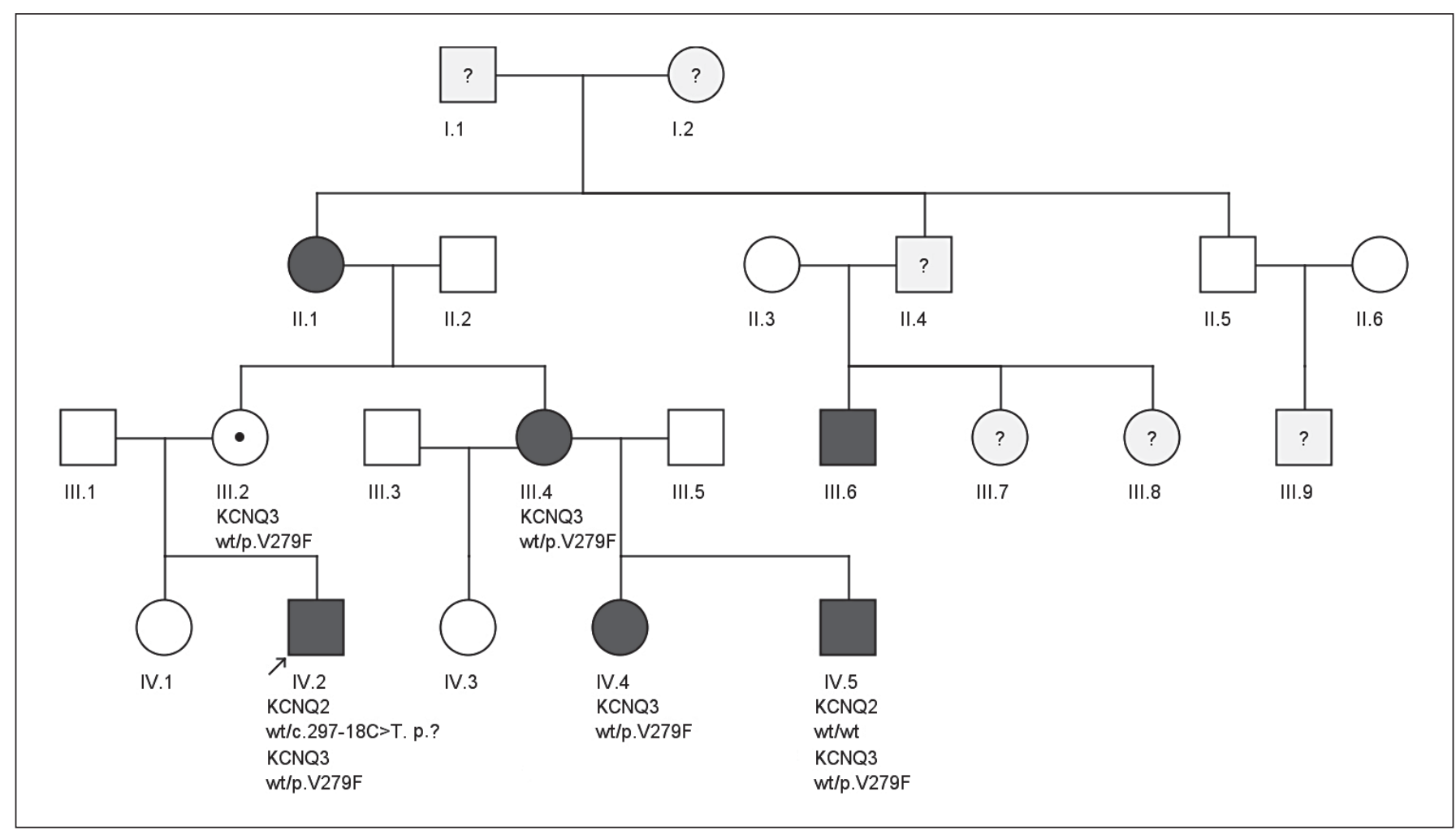

Fig. 2. Pedigree of the family with BFNS. Neonatal convulsions were reported in 6 individuals. The mutation KCNQ3 p.V279F cosegregated with neonatal seizures in all tested individuals and obligate carriers.

cosegregate with the neonatal seizures in patient IV.5 (fig. 2).

\section{Functional Analysis}

KCNQ3 p.V279F replaces the highly conserved amino acid valine at position 279 within the pore-forming transmembrane segment S5 of Kv7.3 channel (fig. 3A, B). To study the functional impact of the mutation we used Xenopus laevis expression system and automated 2-microelectrode voltage clamp technique. In contrast to Kv7.2, homomeric Kv7.3 channels seem to be unstable in heterologous expression systems, which is why we could not examine them separately. Therefore, KCNQ2 cRNA was injected alone or co-injected with the cRNA encoding WT or mutant KCNQ3, and the resulting potassium currents were investigated. Current amplitudes, analyzed at the end of the 2-second depolarizing pulse to $+40 \mathrm{mV}$, were normalized to the mean value obtained for the WT Kv7.2/Kv7.3 heteromers recorded on the same day. As expected, coexpression of Kv7.2 and Kv7.3 WT yielded about 15-fold increased currents compared to the WT
Kv7.2. However, when p.V279F was coexpressed with Kv7.2 WT, the resulting current reached only $~ 12 \%$ of the control Kv7.2/Kv7.3 currents, indicating a clear loss of function ( $\mathrm{p}<0.0001$, one-way ANOVA, fig. 3C). We further observed tentative reduction, but no significant difference compared to the WT Kv7.2/Kv7.3 current amplitudes, when we coexpressed Kv7.2 with Kv7.3 WT and the V279F mutation in a 2:1:1 ratio, mimicking the putative proportion of mutant and WT subunits in an affected individual. Moreover, the voltage dependence of activation of the Kv7.2/Kv7.3/p.V297F was not changed compared to the WT Kv7.2/Kv7.3 channels (fig. 3D).

\section{Discussion}

We identified 6 affected individuals in 3 generations of a family with neonatal convulsions starting between $\mathrm{d} 2$ and d6 (fig. 2). Seizures were mainly focal clonic seizures occurring during wake or sleep over a period of a few days up to 2 weeks. Additional generalized seizures with cya- 
Table 1. Clinical details of family members with reported neonatal seizures

\begin{tabular}{|c|c|c|c|c|c|c|}
\hline Patients & IV.2 (IP) & IV.4 & IV.5 & III. 4 & II.1 & III.6 \\
\hline Mutation & $\begin{array}{l}\text { KCNQ3 c. } 835 \mathrm{G}>\mathrm{T} \text { (het) } \\
\text { p.V279F } \\
\text { KCNQ2 c.297-18C>T } \\
\text { p.? }\end{array}$ & $\begin{array}{l}\text { KCNQ3 c.835G }>\mathrm{T} \\
\text { (het) p.V279F }\end{array}$ & $\begin{array}{l}\text { KCNQ3 c.835G }>\mathrm{T} \\
\text { (het) p.V279F } \\
\text { KCNQ2 wild type }\end{array}$ & $\begin{array}{l}\text { KCNQ3 c. } 835 \mathrm{G}>\mathrm{T} \\
\text { (het) p.V279F }\end{array}$ & not done & not done \\
\hline $\begin{array}{l}\text { Gestational age, } \\
\text { weeks }\end{array}$ & $39+2$ & $39+2$ & $39+0$ & $39+3$ & not known & not known \\
\hline $\begin{array}{l}\text { Seizure onset } \\
\text { (day of life) }\end{array}$ & d5 & d6 & d5 & d3 & during first week & $\begin{array}{l}\text { during first } \\
\text { week }\end{array}$ \\
\hline $\begin{array}{l}\text { Seizure cessation } \\
\text { (day of life) }\end{array}$ & $\mathrm{d} 20$ & $\mathrm{~d} 12$ & $\mathrm{~d} 10$ & $\mathrm{~d} 4$ & not available & not available \\
\hline $\begin{array}{l}\text { Treatment and } \\
\text { outcome }\end{array}$ & $\begin{array}{l}\text { unsuccessful trials with } \\
\text { pyridoxine, pyridoxal- } \\
\text { phosphate, folinic acid, } \\
\text { LEV after d9, slowly } \\
\text { increasing up to } 65 \mathrm{mg} / \mathrm{kg} \\
\text { on d20 (no further seizures } \\
\text { after d20) }\end{array}$ & $\begin{array}{l}\text { LEV after d12, up to } \\
70 \mathrm{mg} / \mathrm{kg} \text { (no further } \\
\text { seizures after first } \\
\text { dose) }\end{array}$ & $\begin{array}{l}\text { LEV after d6, up to } \\
85 \mathrm{mg} / \mathrm{kg} \text { on d8 (still } \\
\text { up to } 4 \text { seizures per } \\
\text { day), } \\
\text { OXC after d10, up to } \\
20 \mathrm{mg} / \mathrm{kg} \text { (no further } \\
\text { seizures after first } \\
\text { dose) }\end{array}$ & no treatment & $\begin{array}{l}\text { no treatment, } \\
\text { spells prompted } \\
\text { cardiological } \\
\text { monitoring and } \\
\text { investigations }\end{array}$ & not available \\
\hline EEG at follow-up & normal & $\begin{array}{l}\text { at } 6 \text { weeks, focal sharp } \\
\text { waves on interictal } \\
\text { EEG, normal EEG } \\
\text { after } 5 \text { months }\end{array}$ & normal & normal & not known & not known \\
\hline $\begin{array}{l}\text { Developmental } \\
\text { outcome }\end{array}$ & normal at 4 years & normal at 2 years & normal & normal & normal & normal \\
\hline Brain imaging & normal MRI at d6 & $\begin{array}{l}\text { normal ultrasound } \\
\text { at } \mathrm{d} 6\end{array}$ & $\begin{array}{l}\text { normal ultrasound } \\
\text { at } \mathrm{d} 6\end{array}$ & not done & not done & not done \\
\hline $\begin{array}{l}\text { Additional } \\
\text { investigations }\end{array}$ & $\begin{array}{l}\text { normal results for tandem- } \\
\text { MS, urinary organic acids, } \\
\text { creatine metabolism; CSF } \\
\text { analysis incl. glucose } \\
\text { quotient, neurotransmitter, } \\
\text { pterines, sulfite test }\end{array}$ & $\begin{array}{l}\text { no additional } \\
\text { investigations }\end{array}$ & $\begin{array}{l}\text { no additional } \\
\text { investigations }\end{array}$ & CSF analysis & not known & not known \\
\hline
\end{tabular}

$\mathrm{CSF}=$ Cerebrospinal fluid; GTCS = generalized tonic-clonic seizures; OFC = occipitofrontal circumference; tandem-MS = tandem mass spectrometry. 


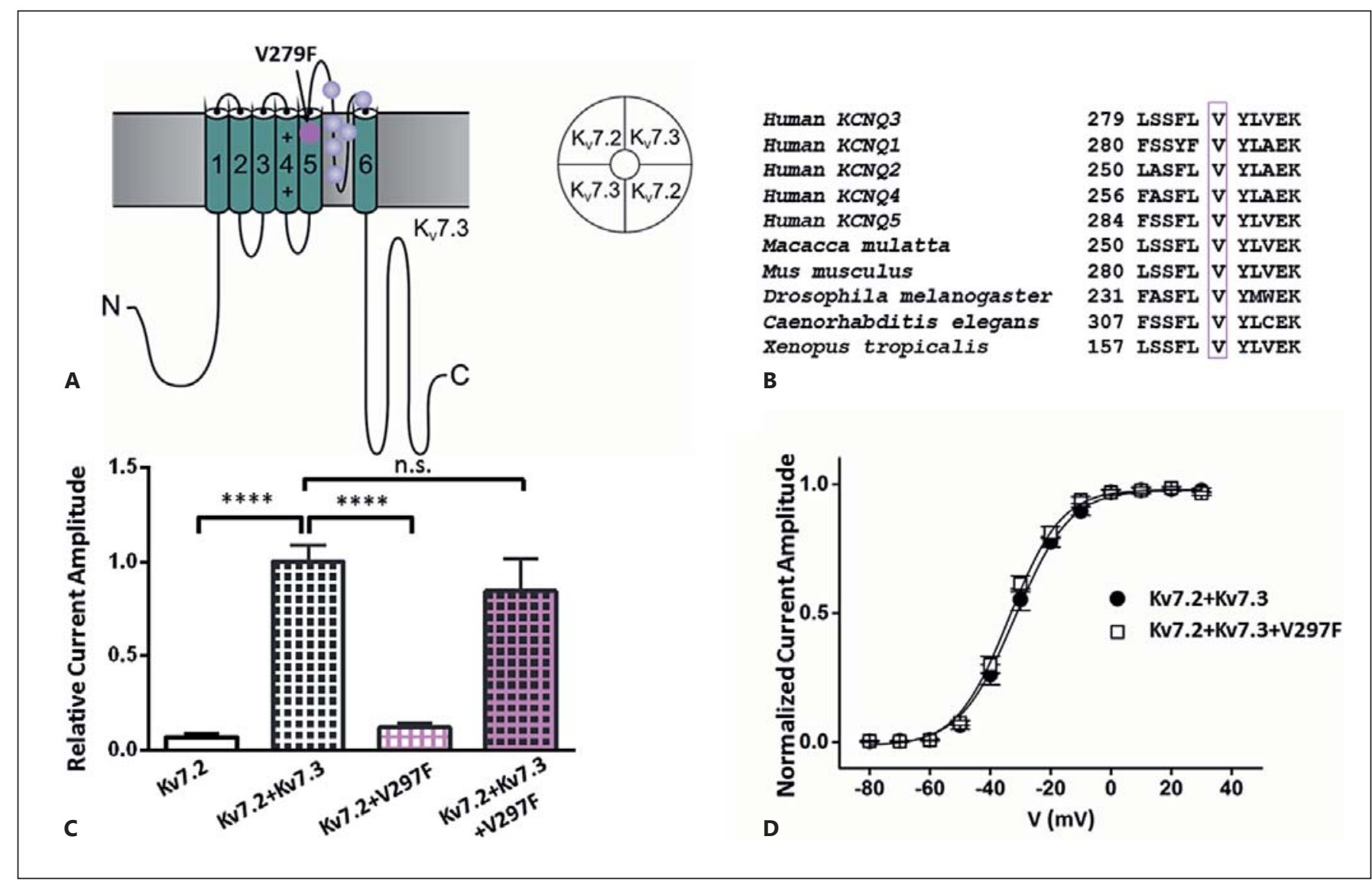

Fig. 3. Functional impact of the Kv7.3 mutation V279F expressed in X. laevis oocytes. A Kv7.3 channel subunit comprises intracellular $\mathrm{N}$ - and C-terminal domains and 6 transmembrane segments, with a pore-forming S5-S6 region. p.V279F predicted localization is within the S5 segment. Previously reported BFNS mutations in the KCNQ3 are located in the pore-forming region [modified after Maljevic and Lerche, 2014]. B p.V279F and the surrounding amino acids show evolutionary conservation. C Expression of WT Kv7.2 and WT Kv7.2 with WT Kv7.3 and/or V279F. Mean \pm SEM of relative current amplitudes analyzed at $+40 \mathrm{mV}$ and normalized to

nosis lasting several minutes prompted treatment in $3 \mathrm{pa}-$ tients (IV.2, IV.4, and IV.5). One child showed a feverassociated seizure after withdrawal of treatment (IV.4), and one individual had another putative seizure during adolescence (III.4), which is in line with reported seizures later in life in members of affected families [Singh et al., 2003; Neubauer et al., 2008].

In the IP, we identified 2 novel variants of unknown significance in KCNQ2 and KCNQ3. The KCNQ2 variant, lying outside the coding sequence, was not predicted to alter splicing and, given it's occurrence in control cohorts, is likely to be a benign polymorphism. Further- the mean of the Kv7.2 $+\mathrm{Kv} 7.3$ currents recorded on the same day were as follows: $0.07 \pm 0.01$ for Kv7.2; $1.0 \pm 0.1$ for Kv7.2 + Kv7.3; $0.11 \pm 0.02$ for Kv7.2 + V279F, and $0.84 \pm 0.07$ for Kv7.2 + V279F $+\mathrm{Kv} 7.3(2: 1: 1) ; \mathrm{n}=7-31{ }^{* * * *} \mathrm{p}<0.0001$ with one-way ANOVA and Dunnett's post hoc test. D Current-voltage relationships of Kv7.2 + Kv7.3 and Kv7.2 + Kv7.3 + V279F (2:1:1) determined from tail current amplitudes. Lines represent fits of a Boltzmann function. $\mathrm{V}_{0.5}$ was $-32 \pm 2(\mathrm{n}=8)$ for Kv7.2 $+\mathrm{Kv} 7.3$ and $-34 \pm 1$ $(\mathrm{n}=9)$ for Kv7.2 + Kv7.3 + V279F (2:1:1).

more, it did not cosegregate with the disease in the family. In contrast, the KCNQ3 variant is predicted to be pathogenic and is absent in the ExAc browser. The variant cosegregated with neonatal seizures in all family members that were available for genetic analysis (fig. 2). As 6 out of 8 known or obligate carriers showed self-limited neonatal seizures, genotype-phenotype analysis of p.V279F suggests incomplete penetrance.

Whereas mutations in KCNQ2 have been identified in the majority of BFNS cases, the number of associated KCNQ3 variants is much smaller: 8 in HGMD or 14 in the KCNQ2/3 database (http://www.rikee.org). Consequent- 
ly, previous functional studies have mainly focused on mutations in KCNQ2 and data on functional consequences of mutated Kv7.3 channels is rare with nonconclusive results [Uhehara et al., 2008, Soldovieri et al., 2014].

We studied the impact of p.V279F in X. laevis oocytes by coexpressing it with the WT Kv7.2 subunits. The coexpression of Kv7.2 with Kv7.3 results in a 10- to 15-fold increase of potassium currents recorded in oocytes, probably due to the increase in the surface expression of the Kv7.2 channels [reviewed in Maljevic and Lerche, 2014]. However, currents obtained from the coexpression of p.V279F with Kv7.2 yielded only about $10 \%$ of the Kv7.2/ Kv7.3 WT current, suggesting that the mutant subunit is either absent, not able to form heteromeric channels, or produces trafficking- or functionally defective heterotetramers with Kv7.2 subunits. Given its position within the pore-forming S5-S6 region of the Kv7.3 channel, the last 2 explanations seem more plausible. If the mutation affects folding of the channel protein, it may not pass the cellular control mechanisms and reach the surface membrane. Alternatively, the mutation may affect channel gating [Maljevic and Lerche, 2014].

In the coexpression experiments supporting the expected expression of mutant and WT subunits in a mutation carrier (Kv7.2:Kv7.3:p.V279F in a 2:1:1 ratio), the observed current reduction was statistically not significant compared to the WT Kv7.2/Kv7.3 coexpression and reached about $15 \%$, instead of the expected $25 \%$ associated with a haploinsufficiency pathomechanism due to a loss of function of 1 of the 4 alleles. The observed tentative reduction of the heteromeric Kv7.2/Kv7.3 currents has been previously seen for some Kv7.2 BFNS mutations in $X$. laevis oocytes [Orhan et al., 2014] and may indicate that the pathophysiological mechanisms in vivo involve other factors not present in our expression system.

So far, haploinsufficiency of Kv7 is known to result in BFNS with only a small number of reported dominant negative missense mutations in KCNQ2 and KCNQ3 [Uhehara et al., 2008; Maljevic and Lerche, 2014]. A dominant negative effect indicates that the presence of the mutant impairs the function of the remaining WT subunits, causing further, more pronounced reduction of potassium currents. The majority of dominant negative mutations in KCNQ2 result in more severe phenotypes with associated neurological disorders such as peripheral nerve hyperexcitability or less favorable seizure outcome and intellectual disability, known as KCNQ2 encephalopathy [Wuttke et al., 2007; Weckhuysen et al., 2013; Orhan et al., 2014]. Interestingly, a recent study also reported mutations in the voltage sensor resulting in a gain of
Kv7.2 channel function associated with a severe epilepsy phenotype [Miceli et al., 2015a]. Only a few reports involved functional characterization of KCNQ3 mutations [Uhehara et al., 2008; Suguira et al., 2009; Soldovieri et al., 2014; Miceli et al., 2015a, b]. The results varied from mere reduction in current amplitudes [Suguira et al., 2009; Soldovieri et al., 2014] over a dominant negative effect associated with BFNS [Uhehara et al., 2008] or with a more severe phenotype with intellectual disability [Miceli et al., 2015b], to the mentioned gain of function in severe epilepsy [Miceli et al., 2015a].

Interestingly, no deletion or truncating mutation has been reported in $K C N Q 3$ to date. In line with this finding, a knockout mouse model is viable, and the lack of KCNQ3 does not produce a specific phenotype [Tzingounis and Nicoll, 2008]. In contrast, homozygous knockin mice carrying a KCNQ3 BFNS-causing mutation develop spontaneous seizures [Singh et al., 2008]. Furthermore, known KCNQ3 mutations seem to cluster within the pore region of the channel (fig. 3A) critical for channel gating. Altogether, this may argue that unlike the KCNQ2, which can cause disease by both haploinsufficiency as well as dominant negative mechanism, the KCNQ3 pathogenicity is based on the impact of single amino acid exchanges on the function of affected channels. Due to the low expression of the Kv7.3 channel in heterologous expression systems, this impact is usually assessed using the Kv7.2/ Kv7.3 heterotetramers. However, also other mechanisms, involving the homomeric Kv7.3 or heteromeric Kv7.5/ Kv7.3 channels may play a role in epileptogenesis. Therefore, further identification of KCNQ3 mutations and functional characterization of their consequences in neuronal environment are necessary to elucidate the role of KCNQ3 in BFNS.

Three individuals were treated for repetitive neonatal seizures. As many seizures were accompanied by apnea and significant desaturation, treating physicians refrained from a conservative treatment and applied antiepileptic drugs (AED). Depicting the outcome of AED treatment in the family members remained challenging. While $2 \mathrm{pa}-$ tients became seizure free during treatment with LEV after $\mathrm{d} 12$ (IV.4) and d20 (IV.2), respectively, patient IV.5 still displayed seizures after introduction of LEV from d6 to $\mathrm{d} 10$. He eventually became free of seizures with introduction of OXC after d10. OXC is of specific benefit in KCNQ2-related epilepsy/encephalopathy [Weckhuysen et al., 2013]. Retigabine, a novel AED, specifically activating Kv7 channels by shifting the voltage sensitivity seems to be an ideal candidate compound for KCNQ2/3-associated seizure disorders [Rundfeldt and Netzer, 2000]. 
However, taking into account both the benign course of disease and the self-limiting character, retigabine and other novel AED with potential adverse drug reactions seem less appropriate in the setting of benign neonatal seizures.

In conclusion, we report on the novel mutation $K C N Q 3$ p.V279F as the cause of neonatal seizures in a family. Functional studies add further evidence for the pathogenicity of p.V279F, demonstrating a loss of channel function. Our study highlights the importance of genetic screening in families with neonatal seizures. The knowledge of a familial mutation can help families and caring neonatologists to avoid unnecessary invasive diagnostic and therapeutic attempts. Further studies on KCNQ3 mutations will help to elucidate the role of KCNQ3 in epileptic disorders.

\section{Acknowledgments}

We thank all patients and family members for their participation in this study. We thank Andrea Schmidt, Marcel Brumme and Jessica Schulz for performing EEG recordings and Janina Bosch for experimental assistance. This study was supported by grants from the German network for rare diseases of the BMBF (Ion NeurONet: 01GM1105A to S.M. and H.L.).

\section{Statement of Ethics}

The use of animals and all experimental procedures were approved by local authorities (Regierungspräsidium Tübingen, Tübingen, Germany).

\section{Disclosure Statement}

The authors have no conflicts of interest to declare.

\section{References}

Biervert C, Schroeder BC, Kubisch C, Berkovic SF, Propping P, et al: A potassium channel mutation in neonatal human epilepsy. Science 279:403-406 (1998).

Charlier C, Singh NA, Ryan SG, Lewis TB, Reus $\mathrm{BE}$, et al: A pore mutation in a novel KQT-like potassium channel gene in an idiopathic epilepsy family. Nat Genet 18:53-55 (1998).

-Maljevic S, Lerche H: Potassium channel genes and benign familial neonatal epilepsy. Prog Brain Res 213:17-53 (2014).

- Miceli F, Soldovieri MV, Ambrosino P, De Maria M, Migliore M, et al: Early-onset epileptic encephalopathy caused by gain-of-function mutations in the voltage sensor of Kv7.2 and Kv7.3 potassium channel subunits. J Neurosci 35:3782-3793 (2015a).

Miceli F, Striano P, Soldovieri MV, Fontana A, Nardello R, et al: A novel KCNQ3 mutation in familial epilepsy with focal seizures and intellectual disability. Epilepsia 56:e15-20 (2015b).

- Neubauer BA, Waldegger S, Heinzinger J, Hahn A, Kurlemann G, et al: KCNQ2 and KCNQ3 mutations contribute to different idiopathic epilepsy syndromes. Neurology 71:177-183 (2008).

Orhan G, Bock M, Schepers D, Ilina EI, Reichel $\mathrm{SN}$, et al: Dominant-negative effects of KCNQ2 mutations are associated with epileptic encephalopathy. Ann Neurol 75:382-394 (2014).
Rett A, Teubel R: Neugeborenenkraempfe im - Sugiura Y, Nakatsu F, Hiroyasu K, Ishii A, Hirose Rahmen einer epileptisch belasteten Familie. Wien Klin Wochenschr 76:609-613 (1964).

Rundfeldt C, Netzer R: The novel anticonvulsant retigabine activates $\mathrm{M}$-currents in Chinese hamster ovary-cells tranfected with human KCNQ2/3 subunits. Neurosci Lett 282:73-76 (2000).

Singh NA, Charlier C, Stauffer D, DuPont BR, Leach RJ, et al: A novel potassium channel gene, $K C N Q 2$, is mutated in an inherited epilepsy of newborns. Nat Genet 18:25-29 (1998).

- Singh NA, Westenskow P, Charlier C, Pappas C, Leslie J, et al: KCNQ2 and KCNQ3 potassium channel genes in benign familial neonatal convulsions: expansion of the functional and mutation spectrum. Brain 126:2726-2737 (2003).

- Singh NA, Otto JF, Dahle EJ, Pappas C, Leslie JD, et al: Mouse models of human KCNQ2 and $K C N Q 3$ mutations for benign familial neonatal convulsions show seizures and neuronal plasticity without synaptic reorganization. J Physiol 586:3405-3423 (2008).

S, et al: Lack of potassium current in W309R mutant KCNQ3 channel causing benign familial neonatal convulsions (BFNC). Epilepsy Res 84:82-85 (2009).

Tzingounis AV, Nicoll RA: Contribution of KCNQ2 and KCNQ3 to the medium and slow after hyperpolarization currents. Proc Natl Acad Sci USA 105:19974-19979 (2008).

Uehara A, Nakamura Y, Shioya T, Hirose S, Yasukochi M, Uehara K: Altered KCNQ3 potassium channel function caused by the W309R pore-helix mutation found in human epilepsy. J Membr Biol 222:55-63 (2008).

Weckhuysen S, Ivanovic V, Hendrickx R, Van Coster R, Hjalgrim $\mathrm{H}$, et al: Extending the KCNQ2 encephalopathy spectrum: clinical and neuroimaging findings in 17 patients. Neurology 81:1697-1703 (2013).

Wuttke TV, Jurkat-Rott K, Paulus W, Garncarek M, Lehmann-Horn F, Lerche H: Peripheral nerve hyperexcitability due to dominant-negative KCNQ2 mutations. Neurology 69:20452053 (2007).

Zara F, Specchio N, Striano P, Robbiano A, Gennaro E, et al: Genetic testing in benign familial epilepsies of the first year of life: clinical and diagnostic significance. Epilepsia 54:425-436 (2013). mar D, Heron B, et al: Novel KCNQ2 and $K C N Q 3$ mutations in a large cohort of families with benign neonatal epilepsy: first evidence for an altered channel regulation by syntaxin-1A. Hum Mutat 35:356-367 (2014). 